\title{
A FORMULA FOR THE BEST LENGTH OF CRANK PINS IN STEAM ENGINES.
}

\author{
By Theron Skeel, C.E.*
}

Any rotating journal being loaded has a tendency to become hot, the work absorbed by the friction of the bearing being changed into heat. If surrounded by a non-conducting material, all of this heat would be stored up within it, and the temperature would increase until the bearing was destroyed. The tendency to become hot decreases with the friction, and any means which reduce the friction also reduce the tendency to heat. These are the use of materials for the rubbing surfaces that slide easily upon each other, aided by a good lubricant.

It is claimed by some mechanics that so long as a film of oil remains between, it matters little of what material the rubbing surfaces are. $\dagger$

There is, however, a limit of pressure, beyond which the lubricant is forced from between the surfaces. This limit is probably never reached in crank-pins, and is certainly above $2000 \mathrm{lbs}$. per square inch of bearing. The bearing upon a crank-pin is less than the projected area of the pin. As an example of the limit of pressure, a mill-stone $48^{\prime \prime}$ in diameter, weighing, with its spindle and gear, 2600 lbs., can be run without excessive heating upon a point $11^{\prime \prime}$ in diameter. The load per square inch of area of the point is $2600 \mathrm{lbs}$. nearly.

In computing the work of friction in a journal let-

$P=$ load upon the journal in lbs.

$d=$ diameter of the journal in inches.

$l=$ length of the journal in inches.

$N=$ number of revolutions per minute.

$\mathcal{J}=$ Joules' equivalent.

$f=$ co-efficient of friction.

The work of friction in foot lbs. per minute:

$$
W=P \cdot f \cdot \frac{\pi d}{12} \mathrm{~N} .
$$

And the amount of heat stored up:

* Iron Age.

$\dagger$ A lecture delivered before the students of the Sterens Institute of Teehnology, by Coleman Sellers, Esq., 1872. 


$$
\frac{W}{J}=\frac{P}{J \cdot} f \cdot \frac{\pi d}{12} N
$$

It is probable that every unit of area of the bearing will dissipate an amount of heat depending upon its condition and independent of the whole area.

Let the number of units of heat dissipated by one square inch of surface on a crank-pin in good order $=q$.

Then, in order that the temperature of the pin may not increase, we have :

Solving for $l$,

$$
\text { l. d. } q .=\frac{W}{J}=\frac{P}{J} f \cdot \frac{\pi d}{12} N \text {. }
$$

$$
l=\frac{P}{J} \frac{f}{d . q} \cdot \pi \frac{d}{12} N=P . N . \frac{\pi f}{12 . d . q .}
$$

make $\frac{p f}{12 . J \cdot q}=\frac{\prime 1}{K}$

$$
l=\frac{P \cdot N}{K}
$$

The above formulæ were first published by Mr. Van Buren in "Strength of Iron Parts of Steam Machinery." He also pointed out that the tendency of the bearing to heat-with good lubricationwas independent of the diameter. From a number of cases in practice he deduced the value of

$$
K=350,000 . \therefore l=\frac{P N}{350,000}
$$

where $P=$ area of piston $\times$ maximum pressure less back pressure.

It is the object of this paper to determine a simpler formula for the length of the pin. As the tendency to become hot depends upon the work done by the friction, it is proportional to the. mean pressure upon the pin exerted during the stroke.

Call the area of the piston $=A$, mean effective pressure $=p^{\prime}$.

$$
\therefore W=p^{\prime} A \frac{\pi d .}{12} N . f
$$

Let $s=$ stroke of piston in inch.

$$
\begin{gathered}
\therefore \frac{p^{\prime} A .2 \text { s. } N .}{12 \times 38,000}=\text { indicated horse-power }=I . H . P . \\
\therefore p^{\prime} A . N=\frac{1 . H . P . \times 12 \times 33,000 .}{2.8 .}
\end{gathered}
$$


Formula for best Length of Crank Pins in Steam Engines. $\quad 37$ substituting and reducing

$$
W=\frac{I . H . P .}{2 . s .} \times \frac{33,000 \times 12}{12} \pi d . f .
$$

Now the amount of heat stored up is

$$
\frac{W}{.}
$$

and as before the amount dissipated is $l . d . q$.

$$
\begin{aligned}
& \therefore W=l d q=\frac{1 . H \cdot P .}{2 s} \times 33,000 \times \frac{\pi \cdot d \cdot f .}{J} \\
& \therefore l=\frac{\text { I. H.P. }}{\text { s. } d .} \times \frac{\pi f}{q} \times \frac{33,000}{J} \\
& \text { let } \frac{\pi f}{q} \times \frac{33,000}{\jmath}=\frac{100}{K^{\prime}} \\
& \text { then } l==\frac{1 . H . P}{s} \cdot \frac{100}{K^{\prime}} \\
& \text { or } K^{\prime}=100 \times \frac{I . H . P .}{\text { s. } l}
\end{aligned}
$$

This notation differs from that used by Mr. Van Buren in that the mean pressure is used in place of the maximum pressure, and the length of the bearing in place of the length of the pin. Most of the engines in his table carried the initial pressure in the cylinder about seven per cent. less than the boiler pressure. Also they cut off at about $\frac{5}{8}$ of the stroke. Therefore the mean pressure was about 9-10 the initial pressure. Also the length of the pin exceeded the length of the bearing about 10 per cent.

Therefore, $K$ for this notation :

$$
=350,000 . \times(0.9 \times 1.1)=350,000 \text {, nearly. }
$$

From Van Buren's formula :

$$
l=\frac{P \prime^{\prime} N}{32,000}=\frac{\text { mean pressure } \times N \times A}{350,000}
$$

From the proposed formula,

$$
l=\frac{\text { mean pressure } \times A \times N \times 2 . \times 100}{33,000 \times K^{\prime} \times S \times 12}
$$


Place them equal to each other and there results :

$$
K^{\prime}=\frac{350,000 \times 100 \times 2}{33,000 \times 12}=177
$$

This formula is particularly applicable to compound engines, for which it is difficult to determine a priori the pressures in the cylinders, while the horse-power can be accurately estimated from the size and proportions of the boilers; or, in the case of a marine engine, from the figure of the ship and the proposed speed.

In this formula it must be borne in mind that $l$ equals the length of the bearing on the pin, and not the length of the pin from face to face of cranks.

From the table of examples given below in practice there may be deduced the following: For a value of $K^{\prime \prime}-$ more than 190 -a stream of water has to be kept upon the pin. From 170 to 190 the pin will require close attention, perfect lubrication, and will be likely to heat with any neglect. From 150 to 170 ordinary care will suffice, while from 130 to 150 represents the best modern practice.

Therefore, the best value of $l$ :

$$
l=\frac{\text { I. H.P.P. }}{130 \times s} \text { to } \frac{\text { I. H. P. }}{150 \times s}
$$

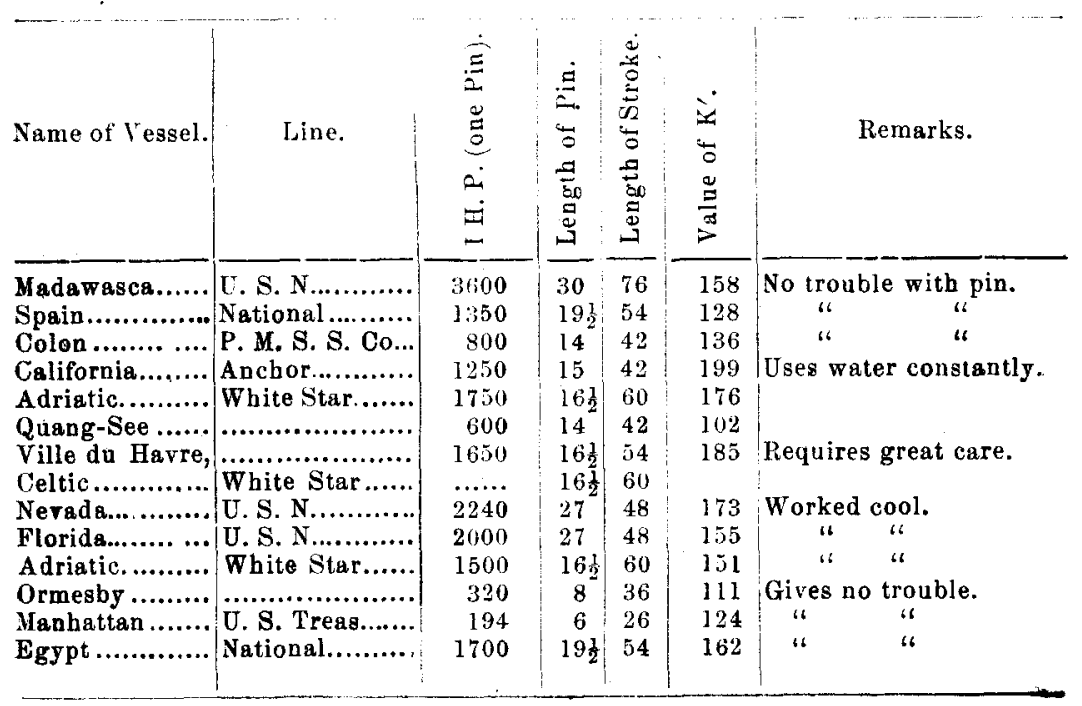

